\title{
Sick Leave
}

National Cancer Institute

\section{Source}

National Cancer Institute. Sick Leave. NCI Thesaurus. Code C148251.

A leave of absence granted because of illness. 